The numbers of professionals being able to support patients and their families will have future challenges. Across Blackpool Fylde and Wyre many patients end up being admitted to the acute setting in circumstances that could have been avoided. The district nursing workforce has seen many changes in our area, with less experienced staff being exposed to a number of clinical conditions that would have been previously managed in the acute setting. The knowledge and understanding around palliative care and its management has become fragmented, with time pressures and workload demands preventing staff from attending training.

We considered how as a hospice and centre for excellence how we could support our community colleagues to enhance their knowledge and skill, to create better links to enable a range of staff to provide 'hospice' care to patients and families in their own homes and to also provide an opportunity for staff to be supported to discuss clinical cases with our CNS Team to enhance shared learning.

The programme of engagement offers bite-size education sessions allowing the opportunity to explore aspects of palliative care in bundles of educational topics. A community based programme that enables a range of staff to focus on a clinical aspect of care, time for reflection, and supported discussion to increase understanding around accurate assessment, knowledge and management of palliative care patients and their families to improve quality of care and patient outcomes.

The programme launched in April 2017. All 13 district nursing teams have signed up, to date there have been 183 staff trained. These results provide proof that as a hospice we can influence care by having a user-friendly based education strategy delivered into the community which could be effectively duplicated in other settings.

\section{P-264 VERIFICATION OF EXPECTED DEATH: A TRAINING INTERVENTION FOR REGISTERED NURSES IN KENT AND SUSSEX}

${ }^{1}$ Nicola Le Prevost, ${ }^{2}$ Mary Kirk, ${ }^{3}$ Jaqueline Joyce. ${ }^{1}$ Pilgrims Hospice, Canterbury, UK; ${ }^{2}$ Kent Community Health Foundation Trust, Kent, UK; ${ }^{3}$ Health Education England, Crawley, UK

\subsection{6/bmispcare-2017-hospice.289}

Background National and local experience with anecdotal reports demonstrate adult end of life care guidance (NICE, 2017) not being achieved for verification of expected death (VoED). Response by Hospice UK collaboratively developing and launching Care After Death publication (2017) new guidance for Registered Nurses (RNs) on VoED. Concern about VoED practice issue at Kent EoLC education meeting resulting in delivering an educational intervention funded by Health Education England shared by local hospices.

Practice Issues Late verification in the community has a distressing impact on families/carers. Contributing factor 'staff are not clear about the legal requirements surrounding the procedure' (Merrifield, 2016).

Aims - Build the confidence and competence of RNs undertaking VoED.

- Align training with Hospice UK guidance.

- Increased number of practitioners with the required skills, knowledge and competence.

- Reducing reliance on medical practitioners.

Methods The education initiative comprises:

- taught face to-face group session of at least three hours.
- completion of a workbook.

- completion of competence assessment.

The workbook has been designed as a support tool to embed learning and provide evidence of reflection and competence. Pre-course and post-course confidence questionnaires are completed by attendees, followed up for post-course attendance.

Findings The pre- and post- course questionnaires indicate a shift towards increased confidence in undertaking VoED. Workbooks have been well received. More detailed findings are not available at the current time due to the project commencement, further data will be available by the time of presentation. Anticipated data will continue to demonstrate increased confidence in RNs. Increased numbers of RNs will be undertaking VoED in the locality.

Conclusion Project demonstrates the highlighting of an area of concern around patient and family experience and addressed, with regional resource support plus collaborative working leading to improving family and carer experience of expected death in the community. With increased numbers of RNs with the confidence and competence to undertake VoED then the NICE guidance should be met.

\section{P-265 DEVELOPING AN INTERDISCIPLINARY POSTGRADUATE PROGRAMME IN PALLIATIVE AND END OF LIFE CARE}

${ }^{1,2}$ Claude Chidiac, ${ }^{3}$ Michael Connolly. 'Saint Francis Hospice, Romford, UK; ${ }^{2}$ London South Bank University, London, UK; ${ }^{3}$ University College Dublin, Dublin, Ireland

10.1136/bmjspcare-2017-hospice.290

Background Interdisciplinary learning is recognised as playing a pivotal role in preparing health and social care professionals in today's complex healthcare environment. Efforts to develop and implement interdisciplinary palliative care education programmes have been scarce and fragmented. Moreover, the World Health Assembly (2014) has adopted a resolution urging all its members to implement palliative care education at specialist and generalist level. In the UK, the end of life care strategy (2008) and subsequent related reports have identified the need for workforce development to improve access and provision of palliative and end of life care.

Aim To design and implement an innovative interdisciplinary curriculum for postgraduate education in palliative care that is person-centred, efficient, accepted, and sustainable.

Methods An interdisciplinary project steering group was established to provide leadership and support, and to oversee the development of the programme. An initial draft of the programme was devised in consultation with the steering group. Three stakeholder events were held in November 2015 which indicated general appreciation and support for an interdisciplinary palliative care education programme at postgraduate level. These events provided feedback on the proposed programme learning outcomes, structure, content, and delivery. A service-user focus group was conducted in early 2016, seeking feedback on draft course content and learning outcomes.

Results Curricular components were redesigned based on feedback from all stakeholders. Openness towards interdisciplinary feedback and external engagement, coupled with flexibility and compromise enabled the development of a person-centred, efficient, accepted and sustainable interdisciplinary programme. Conclusion The development of an interdisciplinary palliative care education programme can encounter multiple barriers; 
however, openness and acceptance of feedback on multiple levels while focusing on learners' needs, to enhance care, can create a successful and feasible programme.

\section{P-266 COGNITIVE BEHAVIOURAL THERAPY (CBT) IN PALLIATIVE CARE: EVALUATION OF STAFF'S KNOWLEDGE, SKILL AND CONFIDENCE FOLLOWING A FOUNDATION LEVEL COURSE}

${ }^{1}$ Kathy Burn, ${ }^{2}$ Louise Lee. ${ }^{1}$ St Christopher's Hospice, London, UKi ${ }^{2}$ St Barnabas Hospice, UK

\subsection{6/bmispcare-2017-hospice.291}

Background How to utilise tools from Cognitive Behavioural Therapy (CBT) 'first-aid' in practice was taught to staff in palliative care settings. This training impacted on their knowledge and confidence when working with distress. Recent studies by Mannix et al. (2006); Anderson (2008); and Moorey et al. (2009) found that palliative care clinicians can benefit from, and effectively use the knowledge, gained from short training courses in CBT.

Aim This three-day St Christopher's, City and Guilds accredited foundation level course was delivered across two hospice sites with a total of 65 staff attending. The staff who attended represented a mix of professions, mainly experienced nursing staff but also occupational therapists, social workers, physiotherapists and counsellors. A 25 question self-reported questionnaire was developed to evaluate changes in staff confidence, knowledge, skill and practical application of psychological support and foundation level CBT. The questionnaires were completed pre- and post- the three- day course by all those attending and again at six months for one cohort.

Results The authors used the Wilcoxon Signed Paired Rank Test (Wilcoxon 1945) to analyse the statistical significance of five of the pre- and post- Visual analogue scale data. The course itself evaluated extremely positively. The results showed that statistically significant improvements at $p>0.05$ in all aspects of knowledge, skill and confidence. Further analysis after six months has also shown sustainable results.

Conclusion Staff reported great benefit from the CBT training, could use the skills and recognised the value of the training in their daily clinical practice. CBT skills can complement previous communication skills training. The training can provide staff with basic skills that can improve knowledge and confidence and can encourage them to adopt a more patient focused and goal centred approach to care.

\section{P-267 IMPLEMENTATION OF EVENING EDUCATION SEMINARS - INSPIRING THE WIDER WORKFORCE}

Helen Harland, Sarah Parnacott. Ashgate Hospicecare, Chesterfield, UK

\subsection{6/bmispcare-2017-hospice.292}

Background We identified the need to educate and up skill colleagues to improve the care of patients across all settings, with a life-limiting diagnosis in North Derbyshire. Challenges in providing education include the need to inspire the workforce, timing of education, staff release and the costs of venues and speakers.

Aims Implementation of free evening, open access education seminars for health and social care professionals across North Derbyshire.
Methods Our day hospice has capacity for 100 people and is now utilised for monthly education. We described our vision to our colleagues in primary and secondary care of providing free education and asked for their assistance to ensure a collaborative approach. We had no budget for speakers and relied on goodwill. We obtain Royal College of Physicians CPD accreditation for the lectures. We do not have to pay for accreditation as the sessions are free and simple refreshments are provided. We are registered with Eventbrite and this provides us with a register and email contact detail of all participants, minimising staff administration costs. Presentations are then emailed to attendees after the event. A certificate of attendance is rewarded on completion of a feedback questionnaire. The feedback is collated and fed back to speakers.

Results Topics presented include: management of end stage heart failure, renal disease, updates in oncological management of breast, bowel and lung cancers, symptom management, and the changing face of palliative care. Attendees include doctors, nurses from diverse care settings, AHPs, and social care staff. Attendance ranges from 43-106 personnel. In 2016-2017 we delivered education to 575 people. Feedback is excellent including staff feeling better equipped to support and manage patients.

Conclusion Evening education seminars are a successful means to educate the wider workforce. Our aim is to continue to build on our success with new and innovative topics into the future.

\section{P-268 IMPACT OF A PALLIATIVE CARE EDUCATION INITIATIVE - LOTHIAN CARE ASSISTANT DEVELOPMENT PROGRAMME}

${ }^{1,2}$ Lyndsay Cassidy, ${ }^{1,2}$ Niall Kieran. ${ }^{1}$ Marie Curie, Edinburgh, UK; ${ }^{2}$ Marie Curie, Glasgow, UK

10.1136/bmjspcare-2017-hospice.293

Introduction Social care teams are increasingly leading the delivery of care for elderly clients and their families who are living with a terminal illness. Marie Curie, in partnership with the City of Edinburgh, East Lothian Council, Midlothian Council and West Lothian Council identified the need for a bespoke education programme to be developed. The Lothian Care Assistant Development Programme encompassed a single study day, followed by an online education programme with mentorship in the participants' work setting.

Aim The overall aim of the project was to increase the knowledge and confidence of frontline staff employed by each of the four Lothian councils to care for clients living with a terminal illness.

Methods A mixed methods approach was used. A survey was completed at four time points, including an impact survey up to six months post completion of the programme. Focus groups and interviews of a sample of social care workers, mentors and managers were also completed.

Results 514 participants completed the single day programme. 71 social care workers continued on to the online module and mentorship programme. Both quantitative and qualitative data will be presented, demonstrating longer-term changes including a team approach to planning and delivering care for people living with a terminal illness. Increased knowledge and confidence from social care staff has resulted in a more person-centred approach to care, prompt recognition of uncontrolled symptoms, and policy change. 